\title{
EL JURÁSICO SUPERIOR EN EL SECTOR NOROCCIDENTAL DE LA PENÍNSULA IBÉRICA (ESPAÑA): BIOESTRATIGRAFÍA Y PRINCIPALES GRUPOS DE BIVALVOS
}

\author{
Graciela DELVENE ${ }^{1}$ Isabel PÉREZ-URRESTI ${ }^{2}$ y \\ Guillermo MELÉNDEZ ${ }^{2}$
}

${ }^{1}$ Museo Geominero, IGME, C/ Ríos Rosas, 23, 28003-Madrid; g.delvene@igme.es
2 Departamento de Ciencias de la Tierra (Paleontología), Universidad de
Zaragoza, c./ Pedro Cerbuna 12, 50009-Zaragoza; isaperez@unizar.es,
gmelende@ unizar.es

Delvene, G., Pérez-Urresti, I. \& Meléndez, G. 2007. El Jurásico Superior en el sector noroccidental de la Península Ibérica (España): bioestratigrafía y principales grupos de bivalvos. [Upper Jurassic at noroccidental area of Iberian Peninsula (Spain): biostratigraphy and main groups of bivalves]. Revista Española de Paleontología, 22 (1), 63-76. ISSN 0213-6937.

\begin{abstract}
Upper Jurassic (upper Oxfordian to lower Kimmeridgian) deposits in the North-western Iberian Range (Aragonese Branch) around the locality of Ricla (Zaragoza) form a thick carbonate and terrigenous marly section showing an increasing content in siliciclastic components. The studied sequence corresponds to the Upper Jurassic Aldealpozo and Sot de Chera Fm. It has been recorded in detail in outcrops $\mathrm{Ri}_{4}$ and $\mathrm{Ri}_{8}$, some 5 to $10 \mathrm{~km}$ North from the village of Ricla. Three lithologic intervals are distinguished: an interval formed by silty to slightly sandy limestones (Aldealpozo Fm); and two intervals of the Sot de Chera Fm, a lower one formed by marls, and an upper one, more siliciclastic and rich in bivalves. The scarce ammonite associations recorded significantly improve the biostratigraphic data, allowing delineate approximately the zone boundaries, from upper Oxfordian Bimammatum Biozone to lower Kimmeridgian (?) Platynota Biozone. The Bimammatum and Hauffianum biozones are represented in the lower, silty to slightly sandy limestone interval (Aldealpozo Fm). The Planula biozone is represented in the marly interval of the Sot de Chera Fm. The former latest Oxfordian Galar Subbiozone, now to be proposed as the basal biozone of the lower Kimmeridgian, is widely developed in the upper part of the sequence. This allows placing the Oxfordian-Kimmeridgian stage boundary roughly coinciding with the lithological transition between both intervals of the Sot de Chera Fm. An updated biostratigraphic scale is, hence, presented according to the recent trends within the Jurasssic Sucommission (ICS; IUGS) about the position of the Oxfordian-Kimmeridgian stage boundary.

Bivalve associations in turn are rich and diversified, and their detail study supplies consistent information to reconstruct sedimentary environmental changes. The analysed assemblage comprises a whole of 2.002 specimens distributed in 47 species and belonging to 32 genera and subgenera. They correspond to the subclasses Palaeotaxodonta (4\%), Pteriomorphia (64,6\%), Palaeoheterodonta (0,5\%), Heterodonta $(29,9 \%)$ and Anomalodesmata (1\%). The most abundant species are Nanogyra nana (J. Sowerby 1822) and Nicaniella (Trautscholdia) carinata (Phillips 1829).
\end{abstract}

Keywords: Ammonites, biostratigraphy, Bivalvia, Iberian Range, Kimmeridgian, Oxfordian, Spain.

\section{RESUMEN}

Los materiales del Jurásico Superior (Oxfordiense superior-Kimmeridgiense inferior) situados al noroeste de la Cordillera Ibérica (Rama Aragonesa), en los alrededores de la localidad de Ricla (Zaragoza), forman una potente sección carbonatada y margosa mostrando un incremento del contenido siliclástico hacia el techo de la serie. La secuencia estudiada corresponde a las formaciones Aldealpozo y Sot de Chera del Jurásico Superior. Se han muestreado detalladamente las secciones de $\mathrm{Ri}_{4}$ y $\mathrm{Ri}_{8}$, ubicadas a 5-10 km al norte de la población de Ricla. Se han diferenciado tres intervalos litológicos: un intervalo formado por calizas limosas ligeramente arenosas (Fm. Aldealpozo); y dos intervalos en la Fm. Sot de Chera, el inferior formado por margas y el intervalo superior más siliciclástico y rico en bivalvos. Las escasas asociaciones de ammonites registradas mejoran significativa- 
mente los datos bioestratigráficos actuales, permitiendo señalar de modo aproximado las zonas desde el Oxfordiense superior (Biozona Bimammatum) al Kimmeridgiense inferior (?) (Biozona Platynota). Las biozonas Bimammatum y Hauffianum están representadas en el intervalo inferior de caliza limosa ligeramente arenosa. La Biozona Planula está representada en el intervalo arenoso de la Fm. Sot de Chera. La antigua Subbiozona Galar del Oxfordiense superior, actualmente propuesta como la biozona basal del Kimmeridgiense inferior, se encuentra ampliamente desarrollada en la parte superior de la secuencia. Esto permite situar el límite Oxfordiense-Kimmeridgiense aproximadamente coincidiendo con la transición litológica entre ambos intervalos de la Fm. Sot de Chera. La escala bioestratigráfica que aquí se presenta está de acuerdo con las actuales tendencias de la Subcomisión de Jurásico (ICS; IUGS) sobre la posición del límite Oxfordiense-Kimmeridgiense.

Las asociaciones de bivalvos muestran una gran abundancia y diversidad en el tramo litológico superior, su análisis detallado proporciona datos consistentes para reconstruir los cambios ambientales y sedimentarios. El estudio realizado comprende un total de 2.002 ejemplares. Se han identificado 47 especies pertenecientes a 32 géneros y subgéneros incluidos en las subclases Palaeotaxodonta (4\%), Pteriomorphia (64,6\%), Palaeoheterodonta (0,5\%), Heterodonta $(29,9 \%)$ y Anomalodesmata (1\%). Las especies más abundantes son Nanogyra nana (J. Sowerby 1822) y Nicaniella (Trautscholdia) carinata (Phillips 1829).

\section{Palabras clave: Ammonites, bioestratigrafía, Bivalvia, Cordillera Ibérica, España, Kimmeridgiense, Oxfor- diense.}

\section{INTRODUCCIÓN}

El Jurásico Superior en el sector noroccidental de la Rama Aragonesa de la Cordillera Ibérica está bien representado en los perfiles situados en las proximidades de Ricla (provincia de Zaragoza; Fig. 1). En el presente trabajo se aborda el estudio paleontológico (Bivalvia) de los materiales del Oxfordiense superior-Kimmeridgiense inferior, representados en las formaciones Aldealpozo y Sot de Chera. Desde el punto de vista de los moluscos bivalvos, su interés radica en la abundancia y diversidad que este grupo taxonómico presenta en la Fm. Sot de Chera en dicho sector.

Los objetivos de este trabajo son: (1) Analizar la sucesión de los materiales del Oxfordiense superior-Kimmeridgiense inferior en los afloramientos $\mathrm{Ri}_{4}$ y $\mathrm{Ri}_{8}$ de Ricla (Zaragoza) incluyendo los nuevos datos bioestratigráficos de ammonoideos obtenidos con posterioridad a los trabajos previos (ver Delvene et al., 1998). (2) Mostrar la distribución los grupos de bivalvos más abundantes en este marco biocronológico, y (3) Discutir los factores ambientales que han podido intervenir en esta distribución.

\section{ESTUDIOS PREVIOS}

Los materiales del Jurásico Superior de este área han sido tratados por diversos autores. Bulard (1972) estudia las calizas con esponjas a las que asigna la edad Oxfordiense medio (Biozona Transversarium) a superior (Biozona Bimammatum) y el resto de materiales del Oxfordiense superior lo encuentra en facies margosas. Sobre las mismas y en los primeros bancos siliciclásticos cita la presencia de Progeronia sp. indicativa del Kimmeridgiense. El área Ricla-Tarazona fue estudiada por Nieva (1986) y la correlación entre este sector y la zona más occidental fue objeto de estudio de Nieva et al. (1986) y posteriormente de Alonso et al. (1989). Meléndez (1989) aporta los primeros datos bioestratigráficos de detalle en el sector de Ricla asignando el techo de la subyacente Fm. Yátova a la Biozona Hypselum y el tramo superior de la Fm. Yátova (de calizas limosas) a las biozonas Bimammatum-Planula.

Aurell (1990) estudia estos materiales desde el punto de vista sedimentológico y define una nueva unidad litoestratigráfica con rango de Miembro en el área de Ricla. Se trata de una unidad arenosa denominada Areniscas y calizas de Ricla, incluida en la Fm. Torrecilla. Esta formación constituye una unidad equivalente lateral de la Fm. Loriguilla en la Sierra de Cameros. Pérez-Urresti $(1995,1996)$ estudia la sucesión de ammonoideos y la bioestratigrafía del Oxfordiense superior de Ricla en el perfil de $\mathrm{Ri}_{4}$. Bádenas (1997) proporciona nuevas precisiones sobre las unidades litoestratigráficas en el sector Ricla-Calatorao y un análisis sedimentológico detallado de las mismas. Delvene (1997) y Delvene et al. (1998) estudian las especies de bivalvos más abundantes en la Fm. Sot de Chera en los Montes de los Picarros. Bádenas (1999) y Bádenas et al. (1999) describen con detalle las unidades litoestratigráficas añadiendo nuevos datos bioestratigráficos sobre las unidades comprendidas entre el Oxfordiense superior y Tithónico inferior en el sector Ricla-Calatorao. La taxonomía de los bivalvos de este sector ha sido tratada por Delvene (2001a y b) y la interpretación paleoecológica de las asociaciones registradas de bivalvos por Delvene (2003). Recientemente, Fernández-López \& Meléndez (2004) aportan nuevas precisiones tafonómicas, taxonómicas y bioestratigráficas sobre el intervalo del límite entre la Fm. Yátova y el tramo suprayacente de calizas limosas (Fm. Aldealpozo) asignándolo a la Biozona Hypselum, Subbiozona Berrense, y caracterizando la asociación de ammonites reconocida como el Taforregistro de profundización avanzada dentro 

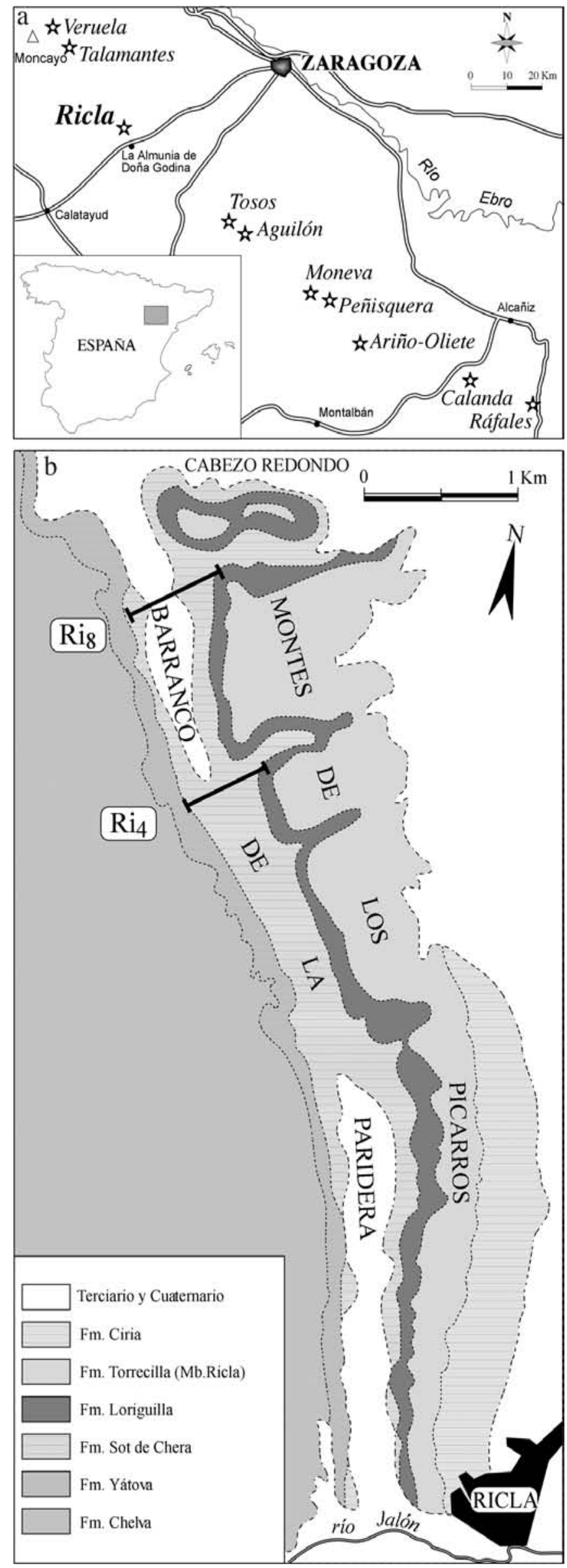

del Hemiciclo de profundización del piso Oxfordiense. Más recientemente, Bello (2005) realiza un nuevo estudio bioestratigráfico, caracterizando el biohorizonte terminal de la Biozona Bifurcatus (Biohorizonte Malinowskae) a techo de la Fm. Yátova en este punto. Ramajo (2006) ha definido y reconocido distintos términos litológicos, entre ellos el Miembro Talamantes dentro de la Fm. Sot de Chera, que abarca las biozonas Hauffianum y Planula.

\section{UNIDADES LITOESTRATIGRÁFICAS}

\section{FORMACIÓN ALDEALPOZO}

La asignación del intervalo litológico estudiado a las unidades litoestratigráficas definidas en la región es actualmente objeto de debate. Este intervalo de calizas limosas a ligeramente arenosas muestra diferencias visibles con la Fm. Sot de Chera en facies típica y constituye en realidad un equivalente lateral de la Fm. Yátova en áreas más orientales de la Cordillera Ibérica. Por estos motivos ha sido incluido dentro de la Fm. Aldealpozo (FernándezLópez \& Meléndez, 2004), una unidad carbonatada-siliciclástica equivalente de la Fm. Yátova en facies de plataforma interna y desarrollada en la parte más occidental de la cuenca, entre el Moncayo y Soria. Bádenas (1999) y Bádenas et al. (1999) la incluyeron en un primer tramo diferenciado de la Fm. Sot de Chera. Recientemente Ramajo (2006) ha propuesto el Mb. Talamantes como una unidad nueva dentro de la Fm. Sot de Chera, en el sector noroccidental de la Rama Aragonesa.

En el presente trabajo el tramo compuesto por calizas limosas a ligeramente arenosas se incluye en la Fm. Aldealpozo, presentando un espesor de 12 a $15 \mathrm{~m}$ y correspondiendo a las biozonas Bimammatum y Hauffianum. Su límite inferior lo constituye el techo de la Fm. Yátova: en ocasiones una superficie de discontinuidad con desarrollo local de superficie ferruginosa que corona los relieves de los niveles bioconstruidos con espongiarios, y lateralmente puede ser una transición gradual. En la base se encuentra un nivel margoso irregular que contiene una asociación

Figura 1. a, Mapa de situación de Ricla (provincia de Zaragoza) en el que se aprecia su posición noroccidental respecto a los principales yacimientos oxfordienses de la Rama Aragonesa de la Cordillera Ibérica. b, Marco geológico del sector de Ricla (provincia de Zaragoza) donde se indica la situación de las secciones $\mathrm{Ri}_{4}$ y $\mathrm{Ri}_{8}$ (modificado de Aurell 1990).

a, Geographic sketch map of the Ricla region (province of Zaragoza) showing its noroccidental position related to the main oxfordian outcrops from the Aragonese Branch of the Iberian Range. b, Geological sketch map of the Ricla region (province of Zaragoza) with position of sections $R i_{4}$ and $R i_{s^{*}}$ (Modified after Aurell 1990). 
condensada con elementos reelaborados de la Biozona Hypselum (subbiozonas Semimammatum y Berrense) por lo que la base de esta unidad correspondería al límite de las biozonas Hypselum y Bimammatum.

\section{FORMACIÓN SOT DE CHERA}

La Formación Sot de Chera es una unidad predominantemente margosa con intercalaciones de margas calcáreas y calizas margosas. En el sector de Ricla los materiales de esta unidad se hacen predominantemente siliciclásticos en la parte superior. El límite inferior, en el área donde fue definida y en el sector central de la plataforma lo constituye la superficie de discontinuidad que corona la Fm. Yátova; en el sector de Ricla que aquí nos atañe su límite inferior es la Fm. Aldealpozo. El límite superior de la Fm. Sot de Chera está formado por una transición gradual hacia la Fm. Loriguilla suprayacente, caracterizada por el incremento progresivo de niveles de caliza, por lo que se sitúa convencionalmente en el punto en que los niveles de caliza comienzan a predominar sobre los intervalos margosos.

La potencia de esta unidad es muy variable, y muestra una disminución progresiva de $\mathrm{NO}$ a SE en la Rama Aragonesa de la Cordillera Ibérica. Los valores máximos, superiores a $150 \mathrm{~m}$ los alcanza en las secciones del sector noroccidental, entre Ricla y Veruela, mientras que en el sector suroriental entre Calanda y Ráfales (provincia de Teruel), no sobrepasa los tres ó cuatro metros de espesor. Se observan incluso diferencias notables entre secciones próximas en el mismo sector: $\mathrm{Ri}_{4}$ es la más potente (cerca de $170 \mathrm{~m})$ y $\mathrm{Ri}_{8}$ aunque es muy próximo presenta aproximadamente $110 \mathrm{~m}$ de la unidad.

Las variaciones litológicas de esta unidad en el sector de Ricla permiten distinguir dos tramos dentro de la misma que se corresponderían con los tramos medio y superior de Bádenas (1997) y Bádenas et al. (1999). El tramo inferior es predominantemente margoso con pequeñas intercalaciones de calizas micríticas. El superior, está formado por una alternancia de calizas y margas arenosas y presenta un contenido claramente mayor en componentes siliciclásticos. El contenido en calizas va siendo progresivamente mayor hacia el techo de la serie, pasando gradualmente a la Fm. Loriguilla.

En conjunto, los materiales de la Fm. Sot de Chera corresponden al intervalo Oxfordiense superior (Biozona Planula)-Kimmeridgiense basal (Biozona Galar). Las dos subzonas clásicas (Planula y Galar) en que se subdividía la Biozona Planula, son admitidas aquí con rango de Biozona, de acuerdo con la tendencia actual en la Subcomisión Internacional de Estratigrafía del Jurásico (ISJS) según la cual la antigua Subbiozona Galar deberá incorporarse con rango de biozona al Kimmeridgiense inferior. El límite Oxfordiense-Kimmeridgiense en las secciones estudiadas se localiza aproximadamente en el tramo superior de esta formación (margas y calizas limosas ligeramente areno- sas). Los escasos datos de ammonoideos permiten asignar el tramo inferior a la Biozona Planula y el superior a la Biozona Galar, si bien los límites de las biozonas, no pueden trazarse con exactitud. El contenido fosilífero, es generalmente bajo en esta unidad, si bien en determinados niveles pueden encontrarse escasos ammonites, braquiópodos y bivalvos. En el sector de Ricla el grupo taxonómico que predomina es el de los bivalvos, destacando su abundancia y diversidad. En estos niveles, aparte de los escasos ammonoideos, están representados también otros grupos de moluscos principalmente belemnites, escafópodos y gasterópodos. Los braquiópodos y equinodermos son minoritarios. A techo del tramo superior, el límite con la Fm. Loriguilla se encuentra subrayado por la presencia de varios bancos de calizas arenosas con frecuentes corales (Bádenas, 1999).

\section{DESCRIPCIÓN DE LOS PERFILES ESTRATIGRÁFICOS}

Las secciones estudiadas, correspondientes a los afloramientos $\mathrm{Ri}_{4}$ y $\mathrm{Ri}_{8}$, se encuentran ubicadas en el término municipal de Ricla, al norte de esta población, en los Montes de los Picarros (Fig. 1b). Los perfiles se han levantado a lo largo de pequeños barrancos en dirección perpendicular a la denominada Rambla de la Paridera. La numeración de los niveles en los perfiles estratigráficos se corresponde con las siglas de campo. La numeración de los perfiles se inicia en la infrayacente Fm. Yátova, de acuerdo con la numeración de los niveles de las columnas estratigráficas realizadas por Fontana \& Meléndez (1990) y Meléndez \& Fontana (1991). La continuación de dichas columnas fue realizada por Delvene (2001a), cuya numeración para las formaciones Aldealpozo y Sot de Chera se siguen en este trabajo y corresponden a las siglas de campo.

\section{PERFIL Ri}

La sección $\mathrm{Ri}_{4}$ es uno de los perfiles de la Rama Aragonesa de la Cordillera Ibérica que ha aportado una mayor abundancia de bivalvos. Los materiales estudiados abarcan desde el techo de la Fm. Yátova hasta la base de la Fm. Loriguilla.

\section{Formación Aldealpozo}

Abarca los niveles 72-100 (Fig. 2a), comprende aproximadamente $10 \mathrm{~m}$ de margas con intercalaciones de calizas limosas, más arenosas hacia el techo del tramo. Estas calizas de color gris oscuro se disponen en bancos tabulares a lenticulares de potencia decimétrica a métrica y baja continuidad lateral. El contenido en ammonites es abundante siendo el nivel 100 el más fosilífero; se han registrado especies de las biozonas Bimammatum y Hauffianum.

El nivel que forma el techo de la Fm. Yátova constituye un intervalo irregular que refleja el carácter bioconstruido de esta unidad (boundstone a bafflestone de espongiarios). En las áreas de relieves positivos el contacto es una superficie irregular, erosiva y localmente encostrada, con aparentes evidencias de hardground. Lateralmente sin embargo, en las áreas deprimidas, en una distancia de decenas de metros, estos relieves pasan a un 

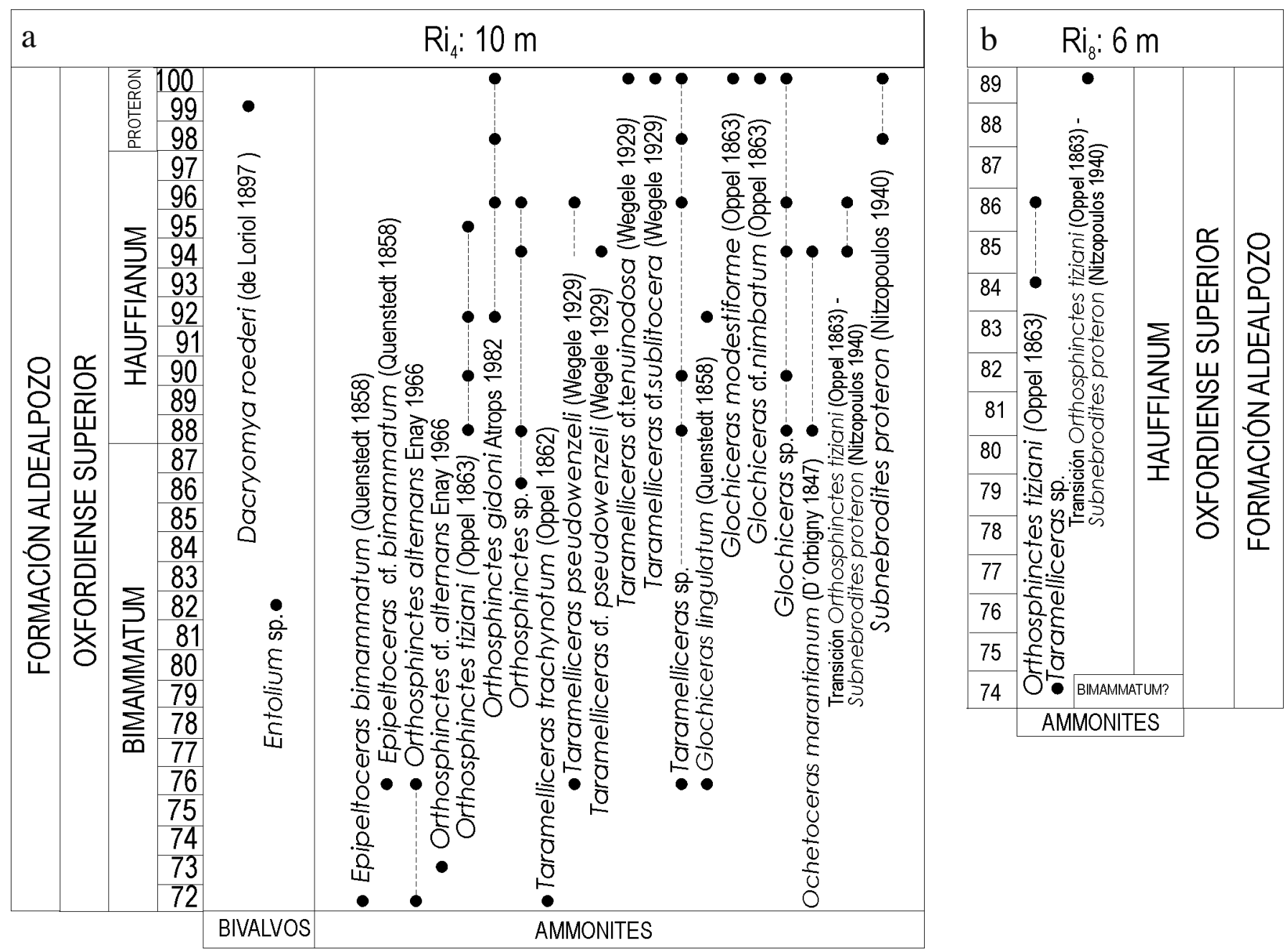

Figura 2. Sucesión de ammonites y bivalvos en los perfiles $\mathrm{Ri}_{4}(\mathbf{a})$ y $\mathrm{Ri}_{8}(\mathbf{b})$ de la Fm. Aldealpozo. Los círculos negros indican que hay registro sin contabilización de ejemplares.

Sequence of bivalves and ammonites at sections $R i_{4}(\boldsymbol{a})$ and $R i_{8}(\boldsymbol{b})$ from Aldealpozo Fm. Black circles show presence of species without a concrete number.

intervalo margoso algo limoso con un alto contenido fosilífero: ammonites abundantes; espongiarios, belemnites, bivalvos y braquiópodos frecuentes; gasterópodos y equinodermos (crinoideos y equinoideos) más escasos. Los ammonites constituyen en su práctica totalidad moldes fragmentados y/o desarticulados; reelaborados, y forman una asociación condensada que incluye elementos característicos del techo de la Biozona Bifurcatus, como Perisphinctes malinowskae Brochwicz-Lewinski 1975, junto con otros de la base de la Biozona Hypselum, como Epipeltoceras semimammatum (Quenstedt 1887), Ochetoceras gr. raixense Fradin 1948, Epipeltoceras berrense (Favre 1876), Orthosphinctes ariniensis (Meléndez 1989) (cf. Fernández-López \& Meléndez, 2004). Los escasos elementos resedimentados constituyen ejemplares incompletos (conchas fragmentadas) de Orthosphinctes gr. kirkdalensis Enay 1966 (non Arkell 1947), que indican la parte superior de la Biozona Hypselum, Biohorizonte "Kirkdalensis".

En el primer nivel situado sobre las calizas con esponjas de Yátova se han reconocido Epipeltoceras bimammatum (Quens- tedt 1858) y Orthosphinctes alternans Enay 1966, que caracterizan la Subbiozona Bimammatum. Esta asociación se mantiene a lo largo de los dos metros siguientes, en los que además se encuentran representantes de la familia Oppeliidae (Subfamilias Glochiceratinae: principalmente Glochiceras modestiforme (Oppel 1863) y Taramelliceratinae; diversos representantes de Taramelliceras gr. costatum (Quenstedt 1887). El primer registro de Orthosphinctes tiziani (Oppel 1863), indicativo de la Biozona Hauffianum, se sitúa hacia la parte media del tramo. A continuación, entre este punto y la parte inferior del siguiente tramo, se han recogido diversas formas intermedias entre las especies Orthosphinctes tiziani (Oppel 1863) y Subnebrodites proteron (Nitzopoulos 1940).

Los bivalvos en el tramo inferior son muy escasos y pertenecen a las familias Nuculidae y Entoliidae.

\section{Formación Sot de Chera}

Primer tramo: Incluye los niveles 101-176, (Fig. 3a). Consta de aproximadamente $100 \mathrm{~m}$ de margocalizas con intercalaciones de 


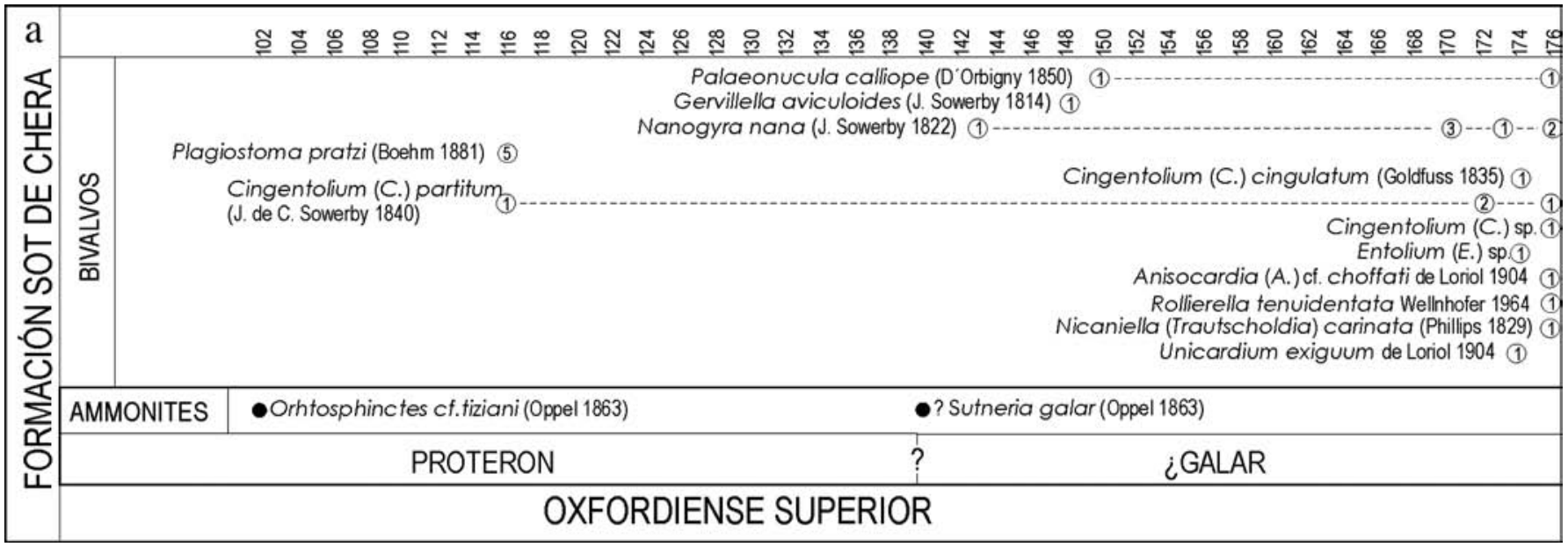

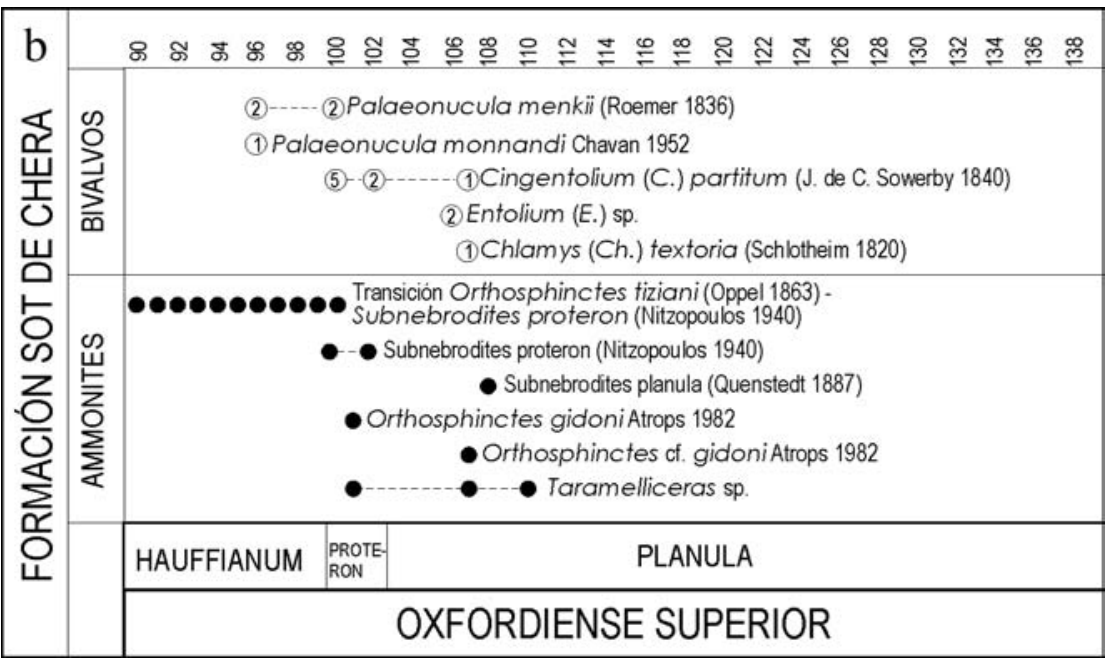

Figura 3. Sucesión de ammonites y bivalvos en los perfiles $\mathrm{Ri}_{4}$ (a) y $\mathrm{Ri}_{8}(\mathbf{b})$ del primer tramo de la Fm. Sot de Chera. Los círculos negros indican que hay registro sin contabilización de ejemplares, los círculos blancos con un número en su interior hacen referencia al número concreto de ejemplares registrados en cada nivel.

Sequence of bivalves and ammonites at sections $R i_{4}(\boldsymbol{a})$ and $R i_{8}(\boldsymbol{b})$ from the first interval of Sot de Chera Fm. Black circles show presence of species without a concrete number while white circles with a number inside, mean the exact number of specimens in each level. calizas limosas, siendo la serie más arenosa hacia la parte superior, donde el registro de bivalvos se hace más frecuente. Los ammonites en este tramo son muy escasos y no han permitido delimitar con precision la Biozona Planula. Entre los bivalvos se encuentran representantes de las subclases Palaeotaxodonta, Pteriomorphia y Heterodonta.

Segundo tramo: Abarca los niveles 177-238, (Fig. 4a), consta de $70 \mathrm{~m}$ de margas con intercalaciones de calizas dispuestas en estratos tabulares. En conjunto el tramo tiene un mayor contenido en siliciclásticos que el anterior. Hacia el techo la relación marga/caliza decrece y la serie se hace progresivamente más carbonatada. Estos materiales se han atribuido a la Biozona Galar. Los bivalvos son muy abundantes en todo el tramo, especialmente en algunos niveles de margocalizas. Están representadas las subclases Palaeotaxodonta, Pteriomorphia, Palaeoheterodonta, Heterodonta y Anomalodesmata. Las especies más abundantes son Gervillella aviculoides (J. Sowerby 1814),
Nanogyra nana (J. Sowerby 1822), Cingentolium (C.) cingulatum (Goldfuss 1835), Cingentolium (C.) partitum (J. de C. Sowerby 1840) y Nicaniella (Trautscholdia) carinata (Phillips 1829). El registro de escafópodos y gasterópodos es abundante en algunos niveles (especialmente 188 y 198) y los belemnites y ammonites son escasos. Los braquiópodos son ocasionales aunque se hacen abundantes en la base de la Fm. Loriguilla que se ha situado en el nivel 238 coincidiendo con el techo de nuestro tramo. Este último nivel está formado por calizas muy arenosas con corales solitarios. En algunos niveles de este intervalo son frecuentes las macroconchas adultas de Ataxiocerátidos propios del techo de la Biozona Galar, especialmente $O r$ thosphinctes gigantoplex (Quenstedt 1887) y en menor medida se registran sus correspondientes microconchas: Orthosphinctes aff. freybergi (Geyer 1961) formadas también por individuos adultos. Se encuentran también representantes de los géneros Taramelliceras (Metahaploceras) sp. y Physodoceras sp. Por

Figura 4. Sucesión de ammonites y bivalvos en los perfiles $\mathrm{Ri}_{4}$ (a) y $\mathrm{Ri}_{8}(\mathbf{b})$ del segundo tramo de la Fm. Sot de Chera. Los círculos negros indican que hay registro sin contabilización de ejemplares, los círculos blancos con un número en su interior hacen referencia al número concreto de ejemplares registrados en cada nivel.

Sequence of bivalves and ammonites at sections $\mathrm{Ri}_{4}(\boldsymbol{a})$ and $\mathrm{Ri}_{8}(\boldsymbol{b})$ from the second interval of Sot de Chera Fm. Black circles show presence of species without a concrete number while white circles with a number inside, mean the exact number of specimens in each level. 

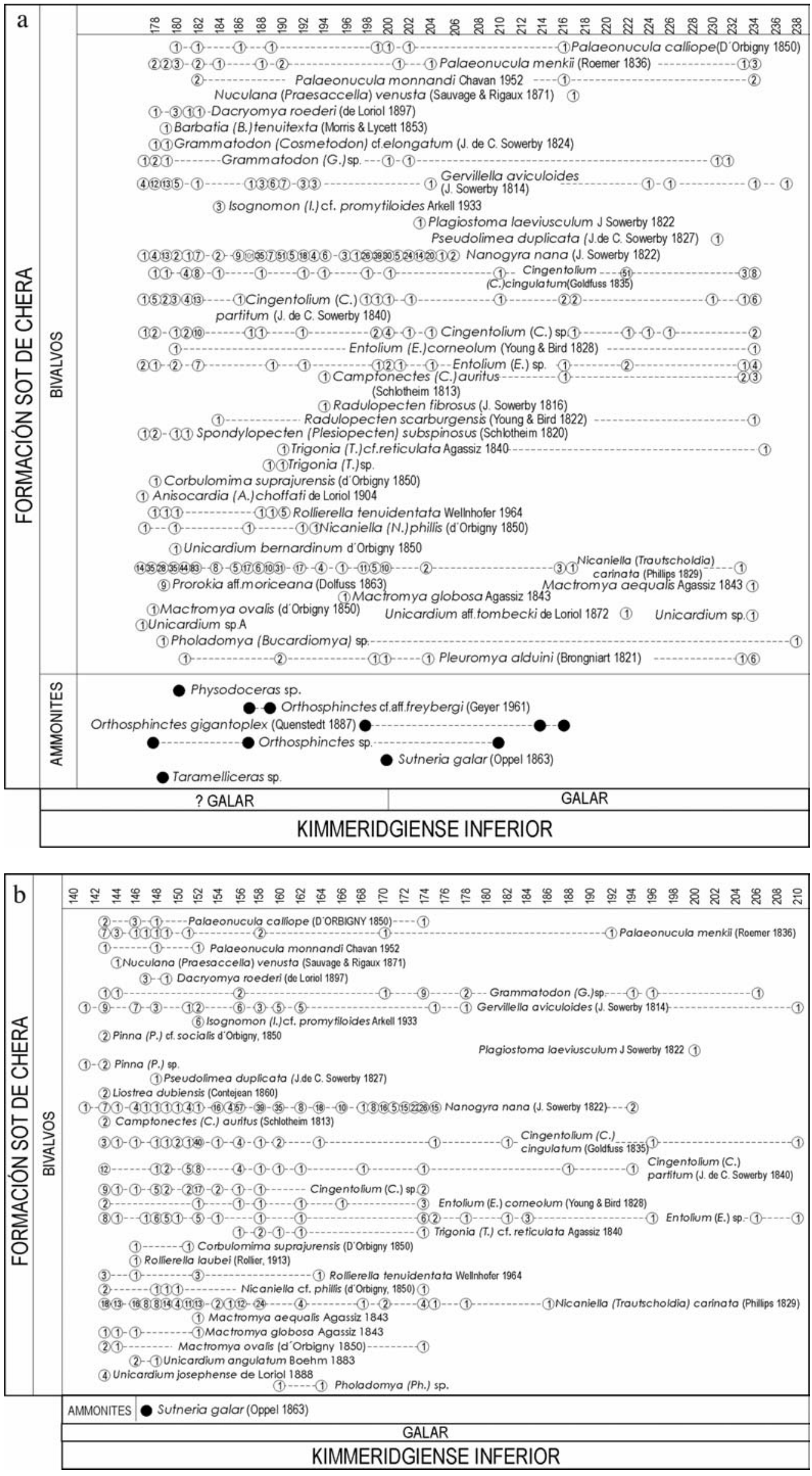
lo general se trata de ejemplares demasiado incompletos y mal conservados, difíciles de determinar. No obstante, a pesar de su carácter escaso y fragmentario, la asociación registrada en este intervalo puede considerarse característica, de un modo general, de la Biozona Galar.

\section{PERFIL Ri ${ }_{8}$}

El perfil denominado $\mathrm{Ri}_{8}$ es paralelo a $\mathrm{Ri}_{4}$ y está situado más al norte, cerca del punto denominado Cabezo Redondo (Fig.1b). Dicho perfil al igual que $\mathrm{Ri}_{4}$ presenta una elevada abundancia de bivalvos y una distribución similar de los mismos. Los materiales estudiados abarcan desde el techo de la Fm. Yátova hasta la base de la Fm. Loriguilla, incluyendo, al igual que en $\mathrm{Ri}_{4}$, las formaciones Aldealpozo y Sot de Chera. En la descripción estratigráfica se han reconocido unos tramos litológicos equivalentes a los establecidos en el trabajo de Bádenas et al. (1999).

\section{Formación Aldealpozo}

Incluye los niveles 74-89, (Fig. 2b), consta de aproximadamente $6 \mathrm{~m}$ de margas con intercalaciones de calizas limosas a ligeramente arenosas. El registro fósil es muy escaso; se han recogido únicamente algunos ejemplares de ammonites de la Biozona Hauffianum, principalmente, Orthosphinctes próximos al grupo de $O$. tiziani (Oppel 1863).

\section{Formación Sot de Chera}

Primer tramo: Abarca los niveles 90-139 (Fig. 3b), son aproximadamente $60 \mathrm{~m}$ de margas con intercalaciones de margocalizas, hacia el techo los niveles más competentes se hacen más arenosos. Algunos niveles presentan escafópodos, crinoides y bivalvos. Entre los últimos, están representadas las familias Nuculidae, Entoliidae y Pectinidae. Los braquiópodos, ammonites y belemnites son más escasos. Entre los niveles 90 y 100 se han registrado formas de ammonites características de las biozonas Hauffianum y Planula. En el nivel 102, algunos ejemplares de Subnebrodites proteron (Nitzopoulos 1940) y en el nivel 108 varios ejemplares de Subnebrodites planula (Quenstedt 1886) que caracterizan en conjunto las dos subbiozonas de la Biozona Planula.

Segundo tramo: Incluye los niveles 140-211 (Fig. 4b), constan de alrededor de $65 \mathrm{~m}$ de margas con intercalaciones de calizas margosas y arenosas en algunas ocasiones dispuestas en estratos tabulares. La relación marga/caliza decrece hacia el techo de la serie, que se hace más arenosa. Es el tramo con mayor abundancia de bivalvos, especialmente entre los niveles 160 y 178. $\mathrm{Al}$ igual que en el tramo superior de $\mathrm{Ri}_{4}$, están representadas las subclases Palaeotaxodonta, Pteriomorphia, Palaeoheterodonta, Heterodonta y Anomalodesmata. Las especies más abundantes son Gervillella aviculoides (J. Sowerby 1814), Nanogyra nana (J. Sowerby 1822), Cingentolium (C.) cingulatum (Goldfuss 1835), Cingentolium (C.) partitum (J. de C. Sowerby 1840) y Nicaniella (Trautscholdia) carinata (Phillips 1829). Entre los niveles 147 y 152 se han registrado ammonites que caracterizan la Biozona Galar: Sutneria galar (Oppel 1863) y diversas formas de Orthosphinctes.

El intervalo que abarca desde el nivel 212 al 224, está formado por calizas muy arenosas y bioclásticas con corales soli-

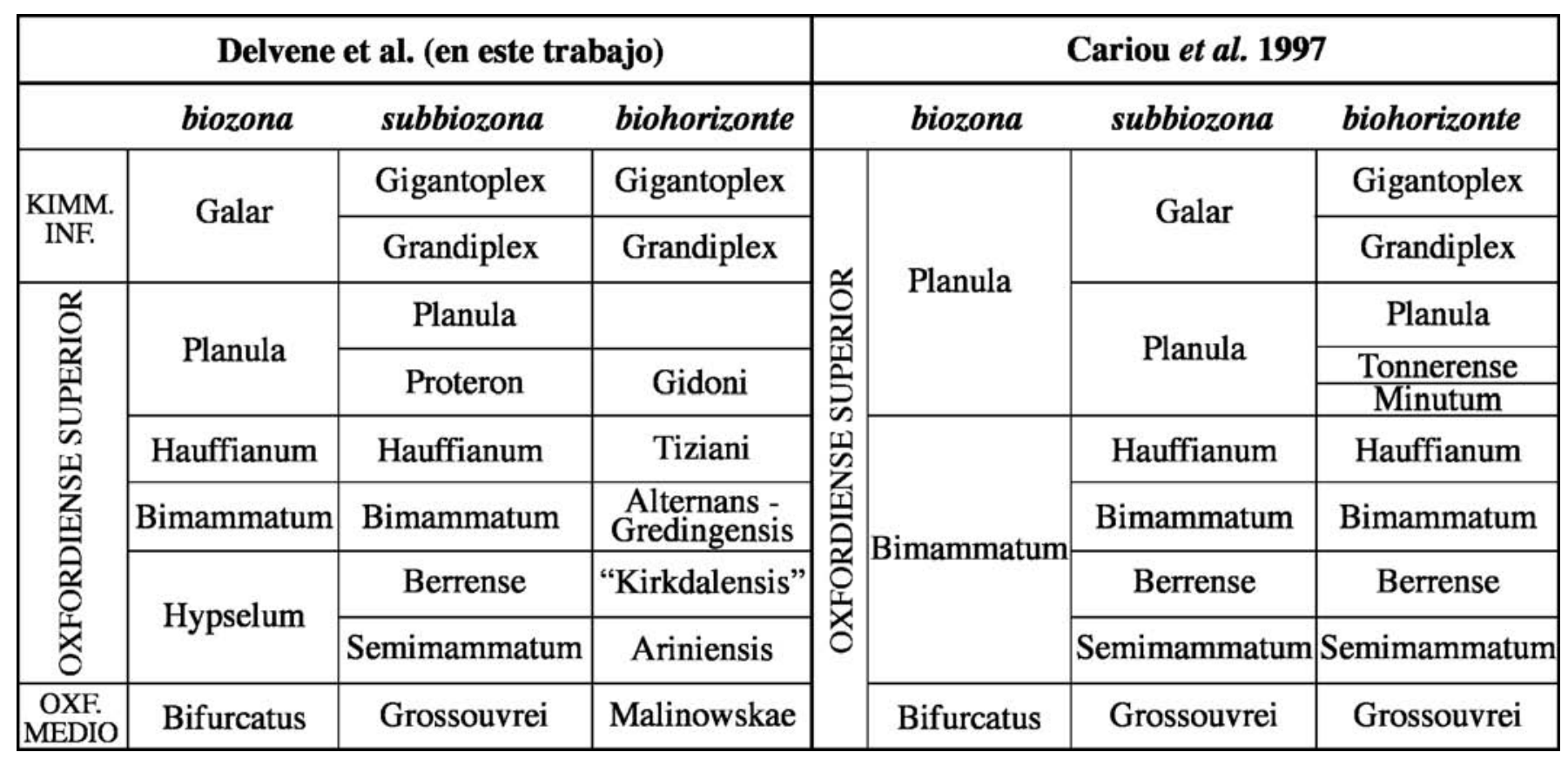

Figura 5. Unidades bioestratigráficas para el Oxfordiense superior-Kimmeridgiense basal de la Cordillera Ibérica (provincia Submediterránea) utilizadas en este trabajo y equivalencia con las definidas por Cariou et al. (1997) para Europa Meridional. Las sucesivas formas de Ataxioceratinae (Orthosphinctes; Planites) han permitido caracterizar y definir en la Cordillera Ibérica los correspondientes biohorizontes para el Oxfordiense superior.

Biostratigraphic units from upper Oxfordian-lower Kimmerdigian from the Iberian Range (Submediterranean province) used in this paper and the equivalent ones definied by Cariou et al. (1997) for Meridional Europe. Sucessive forms of Ataxioceratinae (Orthosphinctes; Planites) have allowed to characterizing and defining lower Oxfordian biohorizons from Iberian Range. 
tarios, braquiópodos y bivalvos. Estos niveles pertenecen a la Fm. Loriguilla y con ciertas dudas se han asignado al Kimmeridgiense inferior, biozonas Platynota-Hypselocyclum aunque los argumentos paleontológicos por el momento son escasos.

\section{BIOESTRATIGRAFÍA}

Los datos expuestos sobre las sucesiones registradas de ammonites permiten confirmar que en los materiales de las formaciones Aldealpozo y Sot de Chera en el sector de Ricla se encuentran representadas todas las unidades bioestratigráficas del Oxfordiense superior reconocidas en la actualidad. Estas incluyen la Biozona Hypselum a techo de la Fm. Yátova; las biozonas Bimammatum y Hauffianum que se encuentran bien desarrolladas en el tramo correspondiente a la Fm. Aldealpozo y han sido caracterizadas por sus taxones típicos, más especialmente por los representantes de los géneros Epipeltoceras Spath 1924, Ochetoceras Haug 1885 y Orthosphinctes Schindewolf 1925. La Biozona Planula se encuentra bien representada en las dos secciones estudiadas dentro del primer tramo de la Fm. Sot de Chera, más potente y predominantemente margoso. La sucesión de formas del género Subnebrodites Spath 1925 ha permitido reconocer y caracterizar dos unidades bioestratigráficas: una inferior (Proteron) y otra superior (Planula) que en este trabajo, de acuerdo con esta discusión, pueden ser reconocidas con status de subbiozona (Fig. 5). El límite preciso entre ellas no puede ser establecido con seguridad por la escasez de ejemplares de ammonoideos. La Biozona Galar (Kimmeridgiense inferior) se puede reconocer por la presencia de Sutneria galar (Oppel 1863) dentro del intervalo superior de la Fm. Sot de Chera. Este intervalo, de carácter más siliciclástico, está formado por tramos de margas con niveles de calizas arenosas. En algunos niveles de este tramo son frecuentes las macroconchas adultas de Ataxiocerátidos propios del techo de esta biozona, especialmente Orthosphinctes gigantoplex (Quenstedt 1887) y, en menor medida, sus correspondientes microconchas: Orthosphinctes aff. freybergi (Geyer 1961) formadas también por individuos adultos. Se encuentran también representantes de los géneros Taramelliceras Del Campana 1904 y Physodoceras Hyatt 1900.

\section{PRINCIPALES GRUPOS DE BIVALVOS}

El muestreo detallado de los perfiles de Ricla ha dado como resultado 2.002 ejemplares de bivalvos, de los cuales 846 pertenecen a la sección $\mathrm{Ri}_{8}$ y 1.156 a la sección $\mathrm{Ri}_{4}$. Las abundancias relativas de bivalvos son similares en ambas secciones, si bien existe una ligera mayor diversidad de especies en $\mathrm{Ri}_{4}$, donde se han registrado 41 especies frente a las 33 reconocidas en $\mathrm{Ri}_{8}$. La comparación entre el registro de bivalvos de ambos perfiles se muestra en las figuras 2-4. El listado de todas las especies reconocidas se recoge en la Tabla 1 . Se incluyen en las siguientes subclases: Palaeotaxodonta (4\%), Pteriomorphia $(64,6 \%)$, Palaeoheterodonta $(0,5 \%)$. Heterodonta $(29,9 \%)$ y Anomalodesmata (1\%). Palaeotaxodonta está representado en su totalidad por el Orden Nuculoida y Pteriomorphia por cinco órdenes: Arcoida, Pterioida, Limoida, Ostreoida y Pectinoida, siendo estos dos últimos los de mayor abundancia, debido especialmente a la presencia de Nanogyra nana (J. Sowerby 1822) en Ostreoida (755 ejemplares, Fig. 6d, e) y de las dos especies de Cingentolium (C.) en Pectinoida (193 ejemplares, Fig. 6h, i). En la Subclase Heterodonta, las especies identificadas se ubican en los órdenes Myoida y Veneroida, siendo este último el que predomina, especialmente debido a la presencia de Nicaniella (Trautscholdia) carinata (Phillips 1829) (Fig. 6m), cuyo registro alcanza 530 ejemplares. Por último, las subclases Palaeoheterodonta y Anomalodesmata están representadas por un único orden cada una: Trigonioida $(0,5 \%)$ y Pholadomyoida (1\%) respectivamente.

\section{OBSERVACIONES TAFONÓMICAS Y PALEOECOLÓGICAS}

La mayoría de los bivalvos del Jurásico Superior de la Cordillera Ibérica son suspensívoros. El grupo de los sedimentívoros alcanza un 4\%, y pertenecen al Orden Nuculoida. Este conjunto de organismos bentónicos, excavadores de fondo blando, someros y móviles están presentes casi exclusivamente en el sector Noroccidental de la Cordillera Ibérica, en las inmediaciones de Ricla, Tosos y Aguilón (todos ellos de la provincia de Zaragoza). Los perfiles de Ricla que aquí presentamos muestran la mayor diversidad y abundancia de bivalvos sedimentívoros de la Cordillera Ibérica. Los ejemplares registrados de Palaeonucula, $\mathrm{Nu}$ culana y Dacryomya, se conservan generalmente como valvas aisladas bien preservadas, también hay algunos ejemplares articulados y escasos moldes internos.

Dentro de los bivalvos suspensívoros, se diferencian infaunales, semi-infaunales y epifaunales. Los bivalvos infaunales son todos ellos excavadores de fondo blando, el 30\% son someros (trigónidos y heterodontos en general) y el $1 \%$ son profundos (pholadómidos). Dentro de los heterodontos, que es un grupo bien representado en cuanto a diversidad y abundancia, predominan los que conservan las dos valvas y están articulados, y la "posición mariposa" (="butterfly position") es muy habitual en varias especies de Mactromya y Unicardium. Abundantes ejemplares de Nicaniella (T.) carinata se han encontrado en posición de crecimiento, la presencia de ejemplares juveniles y adultos muestran una buena representación de su desarrollo ontogénico. Este es uno de ejemplos más evidentes de que las asociaciones registradas en estos niveles de la Fm. Sot de Chera corresponden a asociaciones pa- 
leoecológicas, es decir a grupos de elementos autóctonos y por tanto a restos de una paleocomunidad. La presencia de los pholadómidos es ocasional y siempre se conservan como moldes internos articulados. Los bivalvos semi-infaunales bisados $(5,7 \%)$ están representados por las especies de Grammatodon (Cosmetodon), Gervillella y Pinna. La gran mayoría de ellos se conservan como ejemplares articulados y la posición de crecimiento (= "growthing position") en Gervillella aviculoides y Grammatodon es muy frecuente. El resto de los bivalvos se incluyen en los epifaunales, de los cuales un 3\% pertenece a los bisados (Plagiostoma, Pseudolimea y algunos pectínidos), un $37 \%$ a los cementantes (Orden Ostreoida) y el $18 \%$ a los libres con cierta capacidad natatoria (especies de Cingentolium y Entolium). Nanogyra nana alcanza la casi totalidad de los bivalvos epifaunales cementantes. Su desarrollo onto-

Figura 6. Todos los ejemplares figurados proceden de los materiales de la Fm. Sot de Chera de Ricla (provincia de Zaragoza). Las siglas MPZ corresponden al Museo Paleontológico de la Universidad de Zaragoza donde el material se encuentra depositado.

a: Palaeonucula calliope (D’Orbigny 1850), vista izquierda de ejemplar articulado; Perfil Ricla 4; MPZ 00/744. b: Palaeonucula menkii (Roemer 1836), valva izquierda incluida en una valva derecha fragmentada que no pertenecen al mismo ejemplar (se observa claramente el diferente tamaño entre ellas); Perfil Ricla 8; MPZ 00/781. c: Palaeonucula monnandi Chavan 1952, molde interno de valva derecha; Perfil Ricla 8; MPZ 00/811. d-e: Nanogyra nana (J. Sowerby 1822); d, vista izquierda de ejemplar articulado; e, vista derecha; Perfil Ricla 4; MPZ 97/312. f: Radulopecten fibrosus (J. Sowerby 1816), molde externo de valva derecha; Perfil Ricla 4; MPZ 00/1767. g: Gervillella aviculoides (J. Sowerby 1814), vista derecha de ejemplar articulado; Perfil Ricla 8; MPZ 00/983. h: Cingentolium (Cingentolium) partitum (J. de C. Sowerby 1840), ¿valva izquierda? aislada; Perfil Ricla 4; MPZ 00/2073. i: Cingentolium (Cingentolium) cingulatum (Goldfuss 1835), valva derecha aislada; Perfil Ricla 4; MPZ 00/1892. j: Camptonectes (Camptonectes) auritus (Schlotheim 1883), valva no determinada aislada; Perfil Ricla 8; MPZ 00/1711. k: Rollierella tenuidentata Wellnhofer 1964, vista izquierda de ejemplar articulado en "posición mariposa"; Perfil Ricla 8; MPZ 00/2420. l: Trigonia cf. reticulata Agassiz 1840, valva derecha aislada; Perfil Ricla 4; MPZ 00/2383. m: Nicaniella (Trautscholdia) carinata (Phillips 1829), valva izquierda aislada; Perfil Ricla 4; MPZ 00/2682. n: Prorokia aff. moriceana (Dolfuss 1863), vista derecha de ejemplar articulado;

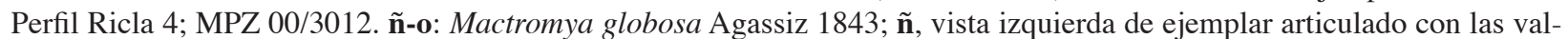
vas entreabiertas; o, vista dorsal; Perfil Ricla 8; MPZ 00/3030. p: Mactromya ovalis (d'Orbigny, 1850), valva izquierda aislada; Perfil Ricla 8; MPZ 00/3035. q-r: Mactromya aequalis Agassiz 1843; q, vista izquierda de molde interno articulado; r, vista dorsal; Perfil Ricla 8; MPZ 00/3028. s: Orthosphinctes alternans Enay 1966, ejemplar con cámara de habitación, el fragmocono está mal conservado; Biozona Bimammatum; Perfil Ricla 4; MPZ 2006/398. t: Orthosphinctes gidoni Atrops 1982, fragmento de cámara de habitación de un ejemplar que conserva el peristoma; Biozona Planula; Perfil Ricla 4; MPZ 2006/399. u: Subnebrodites proteron (Nitzopoulos 1974), fragmento, posiblemente de cámara de habitación, ya que no se observa sutura; el ejemplar ha sufrido una ligera deformación por aplastamiento; Biozona Planula; Perfil Ricla 8; MPZ 2006/400. v: Unicardium angulatum Boehm 1883, vista derecha de ejemplar articulado en "posición mariposa"; Perfil Ricla 8; MPZ 00/3042.

All figured specimens come from levels of Sot de Chera Fm (Ricla, province of Zaragoza). MPZ corresponds to "Palaeontological Museum of University of Zaragoza" where fossils are housed.

$\boldsymbol{a}$ : Palaeonucula calliope (D'Orbigny 1850), left view of articulated specimen; Section Ricla 4; MPZ 00/744. b: Palaeonucula menkii (Roemer 1836), left valve included in a fragmented right valve, both valves do not belong to the same specimen (note the different size of them); Section Ricla 8; MPZ 00/781. c: Palaeonucula monnandi Chavan 1952, internal mould of a right valve; Section Ricla 8; MPZ 00/811. d-e: Nanogyra nana (J. Sowerby 1822); d, left view of articulated specimen; $\boldsymbol{e}$, right view of articulated specimen; Section Ricla 4; MPZ 97/312. $f$ : Radulopecten fibrosus (J. Sowerby 1816); external mould of right valve; Section Ricla 4; MPZ 00/1767. g: Gervillella aviculoides (J. Sowerby 1814), right view of articulated specimen; Section Ricla 8; MPZ 00/983. h: Cingentolium (Cingentolium) partitum (J. de C. Sowerby 1840); isolated left valve?; Section Ricla 4; MPZ 00/2073. i: Cingentolium (Cingentolium) cingulatum (Goldfuss 1835), isolated right valve; Section Ricla 4; MPZ 00/1892. j: Camptonectes (Camptonectes) auritus (Schlotheim 1883), isolated valve; Section Ricla 8; MPZ 00/1711. $\mathrm{k}$ : Rollierella tenuidentata Wellnhofer 1964, left view of articulated specimen in "butterfly position"; Section Ricla 8; MPZ 00/2420. l: Trigonia cf. reticulata Agassiz 1840, isolated right valve; Section Ricla 4; MPZ 00/2383. m: Nicaniella (Trautscholdia) carinata (Phillips 1829), isolated left valve; Section Ricla 4; MPZ 00/2682. n: Prorokia aff. moriceana (Dolfuss 1863), right view of articulated specimen; Section Ricla 4; MPZ 00/3012. $\tilde{\boldsymbol{n}}-\boldsymbol{o}$ : Mactromya globosa Agassiz 1843; $\tilde{\boldsymbol{n}}$, left view of articulated specimen with opened valves; o, dorsal view; Section Ricla 8; MPZ 00/3030. p: Mactromya ovalis (d'Orbigny, 1850), isolated left valve; Section Ricla 8; MPZ 00/3035. q-r: Mactromya aequalis Agassiz 1843; q, left view of an articulated internal mould; $\boldsymbol{r}$, dorsal view; Section Ricla 8; MPZ 00/3028. s: Orthosphinctes alternans Enay 1966, specimen with body chamber; phragmocone is bad preserved; Bimammatum Biozone; Section Ricla 4; MPZ 2006/398. t: Orthosphinctes gidoni Atrops 1982, fragment of body chamber with peristome preserved; Planula Biozone; Section Ricla 4; MPZ 2006/399. u: Subnebrodites proteron (Nitzopoulos 1974), fragment, possibly of body chamber; suture is not observed; the specimen has undergone a slight deformation by crushing; Planula Biozone; Section Ricla 8; MPZ 2006/400. v: Unicardium angulatum Boehm 1883, right view of articulated specimen in "butterfly position"; Section Ricla 8; MPZ 00/3042. 


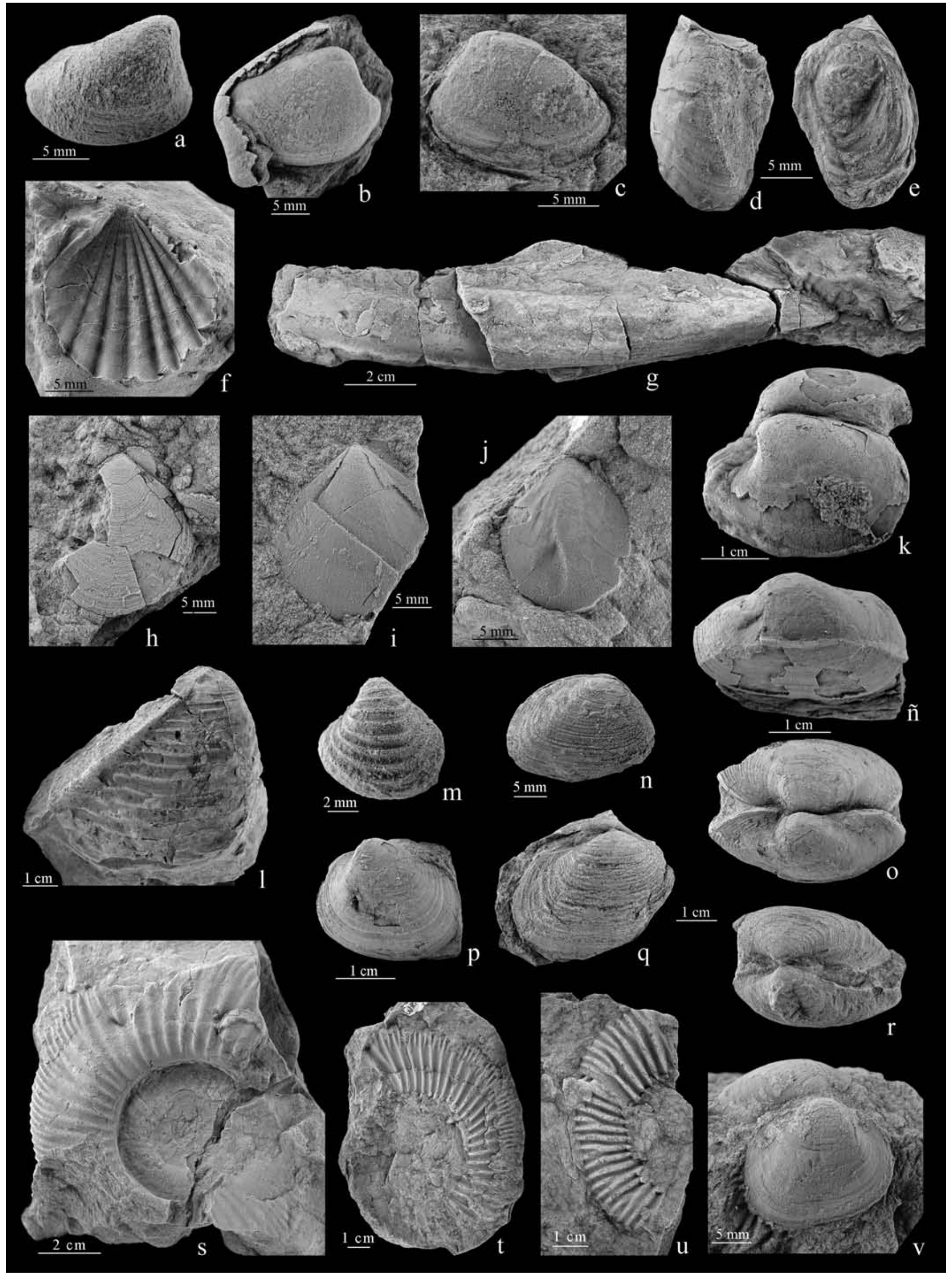




\begin{tabular}{|c|c|}
\hline \multicolumn{2}{|r|}{ Subclase Palaeotaxodonta } \\
\hline Orden Nuculoida (4\%) & $\begin{array}{l}\text { — Palaeonucula calliope (d'Orbigny 1850) (Fig. 6a) } \\
\square \text { - Palaeonucula menkii (Roemer 1836) (Fig. 6b) } \\
\square \text { - Palaeonucula monnandi Chavan 1952 (Fig. 6c) } \\
\square \text { - Nuculana (Praesaccella) venusta (Sauvage \& Rigaux 1871) } \\
\square \text { - Dacryomya roederi (de Loriol 1897) }\end{array}$ \\
\hline \multicolumn{2}{|r|}{ Subclase Pteriomorphia } \\
\hline Orden Arcoida (1,5\%) & $\begin{array}{l}\square \text { Barbatia (Barbatia) tenuitexta (Morris \& Lycett 1853) } \\
\square \text { Grammatodon (Grammatodon) sp. } \\
\square \quad \text { Grammatodon (Cosmetodon) elongatum (J. de C. Sowerby 1824) }\end{array}$ \\
\hline Orden Pterioida (6\%) & $\begin{array}{l}\text { - Gervillella aviculoides (J. SOWERBY 1814) (Fig. 6g) } \\
\square \text { I sognomon (Isognomon) cf. promytiloides Arkell 1933 } \\
\text { - Pinna (Pinna) } \text { cf. socialis D'Orbigny } 1850 \\
\text {-Pinna (Pinna) } \mathrm{sp} .\end{array}$ \\
\hline Orden Limoida $(0,5 \%)$ & $\begin{array}{l}\text { - Plagiostoma laeviusculum J. Sowerby } 1822 \\
\square \quad \text { Plagiostoma pratzi (Boehm 1881) } \\
\square \text { - Pseudolimea duplicata (J. de C. Sowerby 1827) }\end{array}$ \\
\hline Orden Ostreoida $(37,8 \%)$ & $\begin{array}{l}\text { Liostrea dubiensis (Contejean 1860) } \\
\square \text { Nanogyra nana (J. Sowerby 1822) (Fig. } 6 \text { d, e) }\end{array}$ \\
\hline $\begin{array}{l}\text { Orden Pectinoida (18,8\%) } \\
\square \text { Cingentolium }(C \text {.) cingulatum (Goldfuss 1835) } \\
\text { (Fig. 6i) }\end{array}$ & $\square$ - Camptonectes $($ C.) auritus (Schlotheim 1813) (Fig. 6j) \\
\hline $\begin{array}{c}\square \text { Cingentolium }(C .) \text { partitum (J. de C. Sowerby 1840) } \\
\text { (Fig. 6h) }\end{array}$ & - Chlamys (Ch.) textoria (Schlotheim 1820) \\
\hline$\square$ - Cingentolium $(C.) \mathrm{sp}$. & ㅁ Radulopecten fibrosus (J. Sowerby 1816) (Fig. 6f) \\
\hline$\square$ Entolium (E.) corneolum (Young \& Bird 1828) & $\square \quad$ Radulopecten scarburgensis (Young \& Bird 1822) \\
\hline$\square$ Entolium (E.) sp. & $\square \quad$ Spondylopecten (Plesiopecten) subspinosus (Schlotheim 1820) \\
\hline \multicolumn{2}{|r|}{ Subclase Palaeoheterodonta } \\
\hline Orden Trigonioida $(0,5 \%)$ & $\begin{array}{l}\text { 口 Trigonia (Trigonia) cf. reticulata Agassiz } 1840 \text { (Fig. 6l) } \\
\square \quad \text { Trigonia (Trigonia) sp. }\end{array}$ \\
\hline \multicolumn{2}{|r|}{ Subclase Heterodonta } \\
\hline Orden Myoida $(0,2 \%)$ & $\square$ Corbulomima suprajurensis (d'Orbigny 1850) \\
\hline $\begin{array}{l}\text { Orden Veneroida }(29,7 \%) \\
\text { - Mactromya ovalis (d'Orbigny 1850) } \\
\text { (Fig. } 6 \mathrm{p} \text { ) }\end{array}$ & ㅁ Anisocardia (A.) cf. choffati de Loriol 1904 \\
\hline - Unicardium angulatum Boehm 1883 & - Rollierella laubei (Rollier 1913) \\
\hline Unicardium bernardinum d'Orbigny 1850 & 口 Rollierella tenuidentata Wellnhofer 1964 (Fig. 6k) \\
\hline ㅁ Unicardium exiguum de Loriol 1904 & $\square$ - Nicaniella $(N$.$) cf. phillis (d'Orbigny 1850$ ) \\
\hline - Unicardium josephense de Loriol 1888 & $\square$ Nicaniella (Trautscholdia) carinata (Phillips 1829) (Fig. 6m) \\
\hline ㅁ Unicardium aff. tombecki de Loriol 1872 & ㅁ Prorokia aff. moriceana (Dolfuss 1863) (Fig. 6n) \\
\hline$\square$ Unicardium sp. A & $\square$ - Mactromya aequalis Agassiz 1843 (Fig. 6q, r) \\
\hline Unicardium sp. & 口 Mactromya globosa Agassiz 1843 (Fig. 6ñ, o) \\
\hline \multicolumn{2}{|r|}{ Subclase Anomalodesmata } \\
\hline Orden Pholadomyoida (1\%) & $\begin{array}{l}\text { Pholadomya (Bucardiomya) } \mathrm{sp} . \\
\text { - Pholadomya (Pholadomya) sp. } \\
\quad \text { Pleuromya alduini (Brongniart 1821) }\end{array}$ \\
\hline
\end{tabular}

Tabla 1. Especies de bivalvos del Jurásico Superior identificadas en los perfiles $\mathrm{Ri}_{4}$ y $\mathrm{Ri}_{8}$ de Ricla (provincia de Zaragoza). Los cuadrados blancos indican la presencia de la especie en el perfil $\mathrm{Ri}_{4}$; los cuadrados negros indican la presencia de la especie en el perfil $\mathrm{Ri}_{8}$.

Upper Jurassic bivalve species identified in $R i_{4}$ and $R i_{8}$ section from Ricla (province of Zaragoza). White squares show presence of species at the section $R i_{4}$; black squares mean the presence of the species at the section $R i_{8}$. 
génico está bien representado, estando presentes ejemplares juveniles y adultos aunque predominando los de talla media (entre 10 y $15 \mathrm{~mm}$ de diámetro anteroposterior). Se registran como ejemplares articulados, valvas derechas (operculares) y mayoritariamente valvas izquierdas, este amplio registro indica la autoctonía de Nanogyra.

La abundancia (2.002 ejemplares) y diversidad (47 especies) de estos niveles de la Formación Sot de Chera (Oxfordiense Superior y Kimmerdigiense inferior) nos permite reconocer varias asociaciones paleoecológicas descritas en detalle en Delvene (2003). En el sector Noroccidental de la Cordillera Ibérica la mayor potencia de la Fm. Sot de Chera coincide con la mayor abundancia y diversidad de bivalvos de todo el Jurásico Superior, asî como la presencia de bivalvos sedimentívoros. Este grupo de bivalvos está directamente relacionado con la presencia de materia orgánica en el agua, son más abundantes hacia la base de la columna y menos frecuentes e incluso ocasionales hacia techo de la misma. Podemos concluir que la energía del medio va aumentando a lo largo de la serie, así como el conjunto de bivalvos epifaunales. Sin embargo la presencia de semi-infaunales en "posición de crecimiento" y la abundancia de ejemplares de infaunales someros en "posición mariposa" (es decir, que vivieron cerca de la interfase sedimento/agua) son pruebas de que la energía del medio nunca fue elevada. El decrecimiento de la Fm. Sot de Chera hacia el SE de la Cordillera Ibérica, coincide con el decrecimiento de bivalvos del Jurásico Superior, siendo nulo el registro de sedimentívoros en otras áreas de la Cordillera Ibérica. La distribución espacial fue un parámetro determinante en el desarrollo de los bivalvos durante el Oxfordiense Superior y el Kimmeridgiense inferior en el área de Ricla, probablemente debido a que este sector fue el más cercano a la costa y por tanto la disponibilidad de nutrientes provino del continente (Delvene, 2003).

\section{CONCLUSIONES}

Los perfiles $\mathrm{Ri}_{4}$ y $\mathrm{Ri}_{8}$ descritos en este trabajo abarcan la Fm. Aldealpozo y la Fm. Sot de Chera, siendo este sector geográfico donde mayor potencia presenta esta última unidad en toda la Cordillera Ibérica. Las sucesiones registradas de ammonites permiten confirmar que en los materiales de las formaciones Aldealpozo y Sot de Chera en el sector de Ricla se encuentran representadas todas las unidades bioestratigráficas del Oxfordiense superior reconocidas en la actualidad. Estas incluyen la Biozona Hypselum a techo de la Fm. Yátova; las biozonas Bimammatum y Hauffianum en la Fm. Aldealpozo, y la biozona Planula en el primer tramo de la Fm. Sot de Chera. El tramo superior de esta formación se ha asignado a la biozona $\mathrm{Ga}-$ lar del Kimmeridgiense inferior. Los bivalvos registrados en los dos perfiles son similares (846 ejemplares de $\mathrm{Ri}_{8} \mathrm{y}$ $1.156 \mathrm{de}^{\mathrm{Ri}}{ }_{4}$ ) respecto a su abundancia y diversidad. La presencia de sedimentívoros, especialmente en la base de la serie, muestra una acumulación en materia orgánica en el sedimento. Este grupo de bivalvos, perteneciente al orden Nuculoida, sólo está presente en este sector geográfico de la Cordillera Ibérica. Los criterios tafonómicos reconocidos en los grupos de bivalvos, permiten identificar las asociaciones de bivalvos como asociaciones paleoecológicas, perteneciendo el $35 \%$ infaunales, $5,7 \%$ semi-infaunales y $58 \%$ epifaunales. La situación geográfica de Ricla, cerca del continente durante el Oxfordiense superior nos hace pensar que el aporte de nutrientes vendría directamente de esa área fuente (el continente), situación muy favorable para el gran desarrollo de los bivalvos.

\section{AGRADECIMIENTOS}

El trabajo realizado se enmarca en el proyecto CGL2006/10380/ BTE del Ministerio de Educación y Ciencia. Los autores agradecen las revisiones de la Dra. Ana Márquez Aliaga y de un revisor anónimo. Las fotografías han sido realizadas por el Servicio de Fotografía Paleontológica de la Universidad de Zaragoza.

\section{REFERENCIAS}

Alonso, A., Aurell, M., Mas, J. R., Meléndez, A. \& Nieva, S. 1989. Estructuración de las plataformas del Jurásico superior en la zona de enlace entre la cuenca Ibérica y el estrecho de Soria. XII Congreso Español de Sedimentología, 1, 175-178.

Aurell, M. 1990. El Jurásico Superior de la Cordillera Ibérica Central (provincias de Zaragoza y Teruel). Análisis de cuenca. Tesis Doctoral, Universidad de Zaragoza, 510 pp. (inédita).

Bádenas, B. 1997. Caracterización sedimentológica y modelización por ordenador de la rampa carbonatada Kimmeridgiense en Ricla (Zaragoza): implicaciones a escala de cuenca. Tesis de Licenciatura, Universidad de Zaragoza, 120 pp. (inédita).

Bádenas, B. 1999. La sedimentación en las rampas carbonatadas del Kimmeridgiense en las cuencas del Este de la Placa Ibérica. Tesis Doctoral, Universidad de Zaragoza, 256 pp. (inédita).

Bádenas, B., Aurell, M., Pérez-Urresti, I. \& Delvene, G. 1999. Estratigrafía y evolución sedimentaria del Oxfordiense superior-Titónico inferior en Ricla (Zaragoza). Geogaceta, 24, 35-38.

Bello, J., 2005. El Oxfordiense en el sector nororiental de la Cordillera Ibérica: bioestratigrafía y paleontología (Ammonoidea) I: Estratigrafía y Tafonomía; II: Sistemática. Tesis Doctoral, Universidad de Zaragoza, 416 pp. (inédito).

Bulard P. F. 1972. Le Jurassique moyen et supérieur de la Chaine Ibérique sur la bordure du bassin de l'Ebre. Tesis Doctoral, Universidad de Nice, 702 pp.

Delvene, G. 1997. Estudio paleontológico de los bivalvos de la Formación Sot de Chera en Ricla, Zaragoza (Ox- 
fordiense Superior, Cordillera Ibérica). IV Congreso de Jurásico de España. Comunicaciones, 61-62.

Delvene, G. 2001a. Los bivalvos del Jurásico Medio y Superior en la Cordillera Ibérica (España): sistemática y paleoecología. Tesis Doctoral, Universidad de Zaragoza, 192 pp. (inédita).

Delvene, G. 2001b. Middle and Upper Jurassic bivalves from the Iberian Range (Spain). Beringeria, 28, 43-104.

Delvene, G. 2003. Middle and Upper Jurassic bivalve associations from the Iberian Range (Spain). Geobios, 36, 519-531.

Delvene, G., Ramajo, J. \& Pérez-Urresti, I. 1998. Nuevos datos sobre paleontología y bioestratigrafía en la Formación Sot de Chera (Jurásico Superior) de Ricla, España. Coloquios de Paleontología, 49, 73-91.

Fernández-López, S. \& Meléndez, G. 2004. Fossilization of ammonites and sedimentary events in deep environments of carbonate platforms (highest middle Oxfordian to lowest upper Oxfordian, Iberian Range, Spain). Rivista Italiana di Paleontología e Stratigrafia, 110, 219-230.

Fontana, B. \& Meléndez, G. 1990. Caracterización bioestratigráfica de la Biozona Transversarium (Oxfordiense medio) en el sector oriental de la Cordillera Ibérica. Geogaceta, 8, 76-78.

Meléndez, G. 1989. El Oxfordiense en el sector central de la Cordillera Ibérica (provincias de Zaragoza y Teruel). Institución Fernando el Católico-Instituto de Estudios Turolenses, Zaragoza-Teruel, 418 pp.
Meléndez, G. \& Fontana, B. 1991. Sobre la posición estratigráfica de Perisphinctes wartae Bukowski y el desarrollo de la Subbiozona Rotoides (Biozona Transversarium, Oxfordiense medio) en la Cordillera Ibérica nororiental. Geogaceta, 10, 38-42.

Nieva, S. 1986. El Jurásico Superior en el Sector de RiclaTarazona. Sedimentología y Paleogeografía. Tesis de Licenciatura, Universidad de Zaragoza, 110 pp. (inédita).

Nieva, S., Aurell, M. \& Meléndez, A. 1986. El Jurásico Superior en el sector central de la Rama Aragonesa de la Cordillera Ibérica. Sedimentología y Paleogeografía. Acta Geológica Hispánica, 21-22, 373-380.

Pérez-Urresti, I. 1995. Estudio bioestratigráfico y paleontológico basado en Ammonoideos del Oxfordiense Superior en la Rama Aragonesa de la Cordillera Ibérica. Tesis de Licenciatura, Universidad de Zaragoza, 102 pp. (inédita).

Pérez-Urresti, I. 1996. Las sucesiones de Ammonoideos del Oxfordiense Superior en la Cordillera Ibérica nororiental: Nuevos datos bioestratigráficos. Coloquios de Paleontología, 48, 125-145.

Ramajo, J. 2006. Evolución sedimentaria del CallovienseOxfordiense en el sector cental de la Cordillera Ibérica (Rama Aragonesa). Tesis Doctoral, Universidad de Zaragoza, 405 pp. (inédita).

Manuscrito recibido: 27 de Junio 2005 Manuscrito aceptado: 26 de Marzo 2007 